\title{
Acknowledgments to reviewers
}

Editors of the Revista Brasileira de Herbicidas acknowledge the important contribution of the reviewers during the year 2015.

Adriano Jakelaitis

Alberto Leão de Lemos Barroso

Alessandro Guerra Da Silva

Alexandre Ferreira Da Silva

Alexandre Gemelli

Alfredo Junior Paiola Albrecht

Ana Beatriz Rocha De Jesus Passos

Anderson Luis Nunes

André Andres

Andreia Cristina Peres Rodrigues Costa

Antonio Carlos Silva Junior

Antonio Mendes De Oliveira Neto

Arthur Arrobas Martins Barroso

Augusto Guerreiro Fountoura Costa

Augusto Kalsing

Autieres Teixeira Faria

Breno Marques Da Silva

Caio Antônio Carbonari

Camila Ferreira

Carita Liberato do Amaral

Carlos Alberto Mathias Azania

Christiane Augusta Diniz Melo 
Claubert Menezes

Denis Fernando Biffe

Dirceu Agostineto

Edson dos Santos

Eliezer Antonio Gheno

Evander Alves Ferreira

Fabiane Pinto Lamego

Fabiano André Petter

Fabiano Aparecido Rios

Felipe Paolinelli Carvalho

Ferdinando Marcos Lima Silva

Franciele Mariani

Geison Rodrigo Aisenberg

Germani Concenço

Giovanna Larissa Gimenes Cotrick Gomes

Gismael Francisco Perin

Gizelly Santos

Guilherme Braga Pereira Braz

Guilherme Felisberto

Gustavo Antonio Mendes Pereira

Gustavo Rabelo Botrel Miranda

Hudson Kagueyama Takano

Hugo Almeida Dan

Ignacio Aspiazú

João Carlos Madalão

João Guilherme Zanetti Arantes

Joice Fernanda Lübke Bonow 
José Barbosa Santos

José Fernando Jurca Grigolli

Josimar Rodrigues Oliveira

Juliana Roberta Gobi Queiroz

Juliano Costa Da Silva

Julio César Guerreiro

Kassio Ferreira Mendes

Leandro Galon

Leandro Paiola Albrecht

Leandro Pereira Pacheco

Leandro Tropaldi

Leonardo Bianco de Carvalho

Leonardo David Tuffi Santos

Luciana Monteiro Aguiar

Luiz Antonio Koslowski

Luiz Felipe Ramalho De Oliveira

Luiz Henrique Morais Franchini

Marcelo Rodrigues Reis

Marco Antonio Moreira Freitas

Matheus De Freitas Souza

Michel Alex Raimondi

Miler Soares Machado

Miriam Hiroko Inoue

Naiara Guerra

Neumárcio Vilanova Costa

Núbia Maria Correia

Patricia Andrea Monquero 
Patrícia Aparecida De Carvalho Felisberto

Paulo César Timossi

Pedro de Figueiredo Rocha Barbosa Martins

Pedro Jacob Christoffoleti

Renan Cantalice De Souza

Ribas A. Vidal

Ronei Bem

Ronilson de Souza Santos

Rubem Silvério Oliveira Junior

Sérgio Oliveira Procópio

Sidnei Douglas Cavalieri

Sidnei Roberto Marchi

Siumar Pedro Tironi

Theodoro Schneider

Wanderson Souza Rabello

Willians Carrega 